\title{
MAPPINGS PRESERVING APPROXIMATE ORTHOGONALITY IN HILBERT $C^{*}$-MODULES
}

\author{
MOHAMMAD SAL MOSLEHIAN* AND ALI ZAMANI
}

\begin{abstract}
We introduce a notion of approximate orthogonality preserving mappings between Hilbert $C^{*}$-modules. We define the concept of $(\delta, \varepsilon)$ orthogonality preserving mapping and give some sufficient conditions for a linear mapping to be $(\delta, \varepsilon)$-orthogonality preserving. In particular, if $\mathscr{E}$ is a full Hilbert $\mathscr{A}$-module with $\mathbb{K}(\mathscr{H}) \subseteq \mathscr{A} \subseteq \mathbb{B}(\mathscr{H})$ and $T, S: \mathscr{E} \longrightarrow \mathscr{E}$ are two linear mappings satisfying $|\langle S x, S y\rangle|=\|S\|^{2}|\langle x, y\rangle|$ for all $x, y \in \mathscr{E}$ and $\|T-S\| \leq \theta\|S\|$, then we show that $T$ is a $(\delta, \varepsilon)$-orthogonality preserving mapping. We also prove whenever $\mathbb{K}(\mathscr{H}) \subseteq \mathscr{A} \subseteq \mathbb{B}(\mathscr{H})$ and $T: \mathscr{E} \longrightarrow \mathscr{F}$ is a nonzero $\mathscr{A}$-linear $(\delta, \varepsilon)$-orthogonality preserving mapping between $\mathscr{A}$ modules, then

$$
\|\langle T x, T y\rangle-\| T\left\|^{2}\langle x, y\rangle\right\| \leq \frac{4(\varepsilon-\delta)}{(1-\delta)(1+\varepsilon)}\|T x\|\|T y\| \quad(x, y \in \mathscr{E}) .
$$
\end{abstract}

As a result, we present some characterizations of the orthogonality preserving mappings.

\section{INTRODUCTION AND PRELIMINARIES}

An inner product module over a $C^{*}$-algebra $\mathscr{A}$ is a (right) $\mathscr{A}$-module $\mathscr{E}$ equipped with an $\mathscr{A}$-valued inner product $\langle\cdot, \cdot\rangle$, which is $\mathbb{C}$-linear and $\mathscr{A}$ linear in the second variable and has the properties $\langle x, y\rangle^{*}=\langle y, x\rangle$ as well as $\langle x, x\rangle \geq 0$ with equality if and only if $x=0$. An inner product $\mathscr{A}$-module $\mathscr{E}$ is called a Hilbert $\mathscr{A}$-module if it is complete with respect to the norm $\|x\|=\|\langle x, x\rangle\|^{\frac{1}{2}}$. An inner product $\mathscr{A}$-module $\mathscr{E}$ has an " $\mathscr{A}$-valued norm" $|\cdot|$, defined by $|x|=\langle x, x\rangle^{\frac{1}{2}}$. A mapping $T: \mathscr{E} \longrightarrow \mathscr{F}$, where $\mathscr{E}$ and $\mathscr{F}$ are inner product $\mathscr{A}$-modules, is called $\mathscr{A}$-linear if it is linear and $T(x a)=(T x) a$ for all $x \in \mathscr{E}, a \in \mathscr{A}$.

Although inner product $C^{*}$-modules generalize inner product spaces by allowing inner products to take values in an arbitrary $C^{*}$-algebra instead of the $C^{*}$-algebra of complex numbers, but some fundamental properties of inner

2010 Mathematics Subject Classification. 47B49, 46L05, 46L08.

Key words and phrases. Orthogonality preserving mapping, Approximate orthogonality, $(\delta, \varepsilon)$-orthogonality preserving mapping, Inner product $C^{*}$-module. 
product spaces are no longer valid in inner product $C^{*}$-modules. For example, not each closed submodule of an inner product $C^{*}$-module is complemented. Therefore, when we are studying in inner product $C^{*}$-modules, it is always of some interest to find conditions to obtain the results analogous to those for inner product spaces. We refer the reader to [13] for more information on the basic theory of Hilbert $C^{*}$-modules.

Let $\mathbb{B}(\mathscr{H})$ be the $C^{*}$-algebra of all bounded operators acting on a complex Hilbert space $(\mathscr{H},(\cdot, \cdot))$ and $\mathbb{K}(\mathscr{H})$ denote the ideal of compact operators. It is well known that the class of Hilbert $\mathbb{K}(\mathscr{H})$-modules is a well-behaved class of Hilbert $C^{*}$-modules and they share many nice properties with Hilbert spaces. For example, these structures have orthonormal bases and all closed submodules of such modules are complemented. Many properties of Hilbert $C^{*}$-modules over $C^{*}$-algebras of compact operators can be found in [2].

Given two vectors $\eta, \zeta$ in a Hilbert space $\mathscr{H}$, we shall denote by $\eta \otimes \zeta \in$ $\mathbb{K}(\mathscr{H})$ the one-rank operator defined by $(\eta \otimes \zeta)(\xi)=(\xi, \zeta) \eta$. Obviously, $\|\eta \otimes \zeta\|=\|\eta\|\|\zeta\|$ and $\operatorname{tr}(\eta \otimes \zeta)=(\eta, \zeta)$. Observe that $\eta \otimes \eta$ is the orthogonal projection to the one dimensional subspace spanned by the unit vector $\eta$. If $T$ is an arbitrary bounded operator on $\mathscr{H}$, then $(\eta \otimes \eta) T(\eta \otimes \eta)=(T \eta, \eta) \eta \otimes \eta$. This shows that $\eta \otimes \eta$ is a minimal projection. Recall that a projection (i.e., a self-adjoint idempotent) $e$ in a $C^{*}$-algebra $\mathscr{A}$ is called minimal if $e \mathscr{A} e=\mathbb{C} e$.

Now let $\mathscr{E}$ be an inner product (respectively, Hilbert) $\mathscr{A}$-module, where $\mathbb{K}(\mathscr{H}) \subseteq \mathscr{A} \subseteq \mathbb{B}(\mathscr{H})$. Let $e=\eta \otimes \eta$ for some unit vector $\eta \in \mathscr{H}$, be any minimal projection. Then $\mathscr{E}_{e}=\{x e: x \in \mathscr{E}\}$, is a complex inner product (respectively, Hilbert) space contained in $\mathscr{E}$ with respect to the inner product $(x, y)=\operatorname{tr}(\langle x, y\rangle), x, y \in \mathscr{E}_{e}$; see [2]. It is not hard to see that $\langle x, y\rangle=(x, y) e$. Note that if $x \in \mathscr{E}_{e}$, then $\|x\|_{\mathscr{E}_{e}}=\|x\|_{\mathscr{E}}$, where the norm $\|\cdot\|_{\mathscr{E}_{e}}$ comes from the inner product $(\cdot, \cdot)$. This enables us to apply Hilbert space theory by lifting results from the Hilbert space $\mathscr{E}_{e}$ to the whole $\mathscr{A}$-module $\mathscr{E}$.

The orthogonality equation and the related orthogonality preserving property play an important role in Hilbert $C^{*}$-modules, operator algebras, $K$-theory and group representation theory; see [1, 3, 8, 11] and the references therein.

Recall that vectors $\eta, \zeta$ in an inner product $\mathscr{H}$ are said to be orthogonal, and write $\eta \perp \zeta$, if $(\eta, \zeta)=0$ and, for a given $\delta \geq 0$, they are approximately orthogonal or $\delta$-orthogonal, denoted by $\eta \perp^{\delta} \zeta$, if $|(\eta, \zeta)| \leq \delta\|\eta\|\|\zeta\|$. For $\delta \geq 1$, it is clear that every pair of vectors are $\delta$-orthogonal, so the interesting case is when $\delta \in[0,1)$. 
A mapping $T: \mathscr{H} \rightarrow \mathscr{K}$, where $\mathscr{H}$ and $\mathscr{K}$ are inner product spaces, is called orthogonality preserving if $\eta \perp \zeta \Rightarrow T \eta \perp T \zeta(\eta, \zeta \in \mathscr{H})$. It is known that orthogonality preserving mappings may be nonlinear and discontinuous but under additional assumption of linearity, a mapping $T$ is orthogonality preserving if and only if it is a scalar multiple of an isometry, that is $T=\gamma U$, where $U$ is an isometry and $\gamma \geq 0$; see [4]. It should be noticed that the same result is obtained in [20] by using a different approach. The orthogonality preserving mappings have been considered also in [15].

Analogously, for $\delta, \varepsilon \in[0,1)$, a mapping $T: \mathscr{H} \rightarrow \mathscr{K}$ is said to be approximately orthogonality preserving, or $(\delta, \varepsilon)$-orthogonality preserving, if $\eta \perp^{\delta} \zeta \Rightarrow T \eta \perp^{\varepsilon} T \zeta(\eta, \zeta \in \mathscr{H})$. Approximately orthogonality preserving mappings have been recently intensively studied in connection with functional analysis and operator theory; cf. [4, 6, 10, 16, 17, 19, 20].

An interesting question is whether a $(\delta, \varepsilon)$-orthogonality preserving mapping $T$ must be close to a linear orthogonality preserving mapping.

In the case where $\delta=0$, Chmieliński [4] and Turnšek [16] verified the properties of mappings that preserve approximate orthogonality in inner product spaces. Also Kong and Cao [10] studied stability of approximate orthogonality preserving mappings and the orthogonality equations. Approximate orthogonality preserving mappings between inner product spaces have been recently considered by Wójcik in [17].

Other approximate orthogonalities in general normed spaces along with the corresponding approximately orthogonality preserving mappings have been studied in $[7,14,18]$. Similar investigations have been carried out in Hilbert spaces in $[5,6,12]$.

It is natural to explore the orthogonality preserving mappings between inner product $C^{*}$-modules. So, a mapping $T: \mathscr{E} \rightarrow \mathscr{F}$, where $\mathscr{E}$ and $\mathscr{F}$ are inner product $\mathscr{A}$-modules, is called orthogonality preserving if $x \perp y \Rightarrow T x \perp$ Ty $(x, y \in \mathscr{E})$. Also, for $\delta, \varepsilon \in[0,1)$, it is called approximately orthogonality preserving, or $(\delta, \varepsilon)$-orthogonality preserving, if

$$
\|\langle x, y\rangle\| \leq \delta\|x\|\|y\| \Rightarrow\|\langle T x, T y\rangle\| \leq \varepsilon\|T x\|\|T y\| \quad(x, y \in \mathscr{E}) .
$$

The natural problems are to describe such a class of approximately orthogonality preserving mappings and whether each $(\delta, \varepsilon)$-orthogonality preserving mapping has to be approximated by an orthogonality preserving mapping. 
Ilišević and Turnšek [9] studied approximate orthogonality preserving mappings on $\mathscr{A}$-modules with $\mathbb{K}(\mathscr{H}) \subseteq \mathscr{A} \subseteq \mathbb{B}(\mathscr{H})$ in the case when $\delta=0$. Orthogonality preserving mappings have been treated also by Frank et al. [8] and Leung et al. [11].

In this paper, we study $(\delta, \varepsilon)$-orthogonality preserving mappings between Hilbert $\mathscr{A}$-modules, which generalize some results from $[4,9,10,16,17]$. In Section 2, some sufficient conditions for a linear mapping to be $(\delta, \varepsilon)$ orthogonality preserving are given. In particular, we show that if $\mathscr{E}$ is a full Hilbert $\mathscr{A}$-module with $\mathbb{K}(\mathscr{H}) \subseteq \mathscr{A} \subseteq \mathbb{B}(\mathscr{H})$ and $T, S: \mathscr{E} \longrightarrow \mathscr{E}$ are two linear mappings such that $|\langle S x, S y\rangle|=\|S\|^{2}|\langle x, y\rangle|$ for all $x, y \in \mathscr{E}$ and $\|T-S\| \leq \theta\|S\|$ with $\theta \in[0,1)$, then $T$ is a $(\delta, \varepsilon)$-orthogonality preserving mapping, where $\varepsilon=\frac{\theta^{2}+2 \theta+\delta}{(1-\theta)^{2}}$.

In Section 3 we prove if $\mathbb{K}(\mathscr{H}) \subseteq \mathscr{A} \subseteq \mathbb{B}(\mathscr{H})$ and $T: \mathscr{E} \longrightarrow \mathscr{F}$ is a nonzero $\mathscr{A}$-linear $(\delta, \varepsilon)$-orthogonality preserving mapping between $\mathscr{A}$-modules, then

$$
\|\langle T x, T y\rangle-\| T\left\|^{2}\langle x, y\rangle\right\| \leq \frac{4(\varepsilon-\delta)}{(1-\delta)(1+\varepsilon)}\|T x\|\|T y\| \quad(x, y \in \mathscr{E}) .
$$

As a result, we obtain some characterizations of the orthogonality preserving mappings in inner product $\mathscr{A}$-modules. Particularly, we show that a nonzero $\mathscr{A}$-linear mapping $T$ is orthogonality preserving if and only if $T$ is $(\varepsilon, \varepsilon)$-orthogonality preserving. Our results improve some theorems due to Chmieliński [4] and Wójcik [17].

\section{Approximate orthogonality preserving property in Hilbert $C^{*}$-MODULES}

In this section, we give some sufficient conditions for a linear mapping to be $(\delta, \varepsilon)$-orthogonality preserving. Recall that the minimum modulus $[T]$ of a linear map $T$ is defined by $[T]:=\inf \{\|T x\|:\|x\|=1\}$.

Proposition 2.1. Let $\theta \geq 1, \lambda \in\left[0, \frac{1}{4}\right)$ and $0 \leq \delta<\frac{1-4 \lambda}{\theta^{4}}$. Let $\mathscr{E}$ and $\mathscr{F}$ be two inner product $\mathscr{A}$-modules and let $T, S: \mathscr{E} \longrightarrow \mathscr{F}$ be nonzero linear mappings such that

(i) $\|T x-S x\| \leq \lambda\|S x\|$ for all $x \in \mathscr{E}$

(ii) $\frac{1}{\theta^{2}} \gamma^{2}\|\langle x, y\rangle\| \leq\|\langle S x, S y\rangle\| \leq \theta^{2} \gamma^{2}\|\langle x, y\rangle\|$ for all $x, y \in \mathscr{E}$,

with some $\gamma \in[[S],\|S\|]$. Then $T$ is a $(\delta, \varepsilon)$-orthogonality preserving mapping, where $\varepsilon=\frac{\lambda^{2}+2 \lambda+\theta^{4} \delta}{(1-\lambda)^{2}}$. 
Proof. It follows from (i) that

$$
\|S x\|=\|S x-T x+T x\| \leq\|S x-T x\|+\|T x\| \leq \lambda\|S x\|+\|T x\| \quad(x \in \mathscr{E}) .
$$

Hence

$$
\|S x\| \leq \frac{1}{1-\lambda}\|T x\| \quad(x \in \mathscr{E}) .
$$

Put $y=x$ in (ii) to get $\|x\| \leq \frac{\theta}{\gamma}\|S x\|$, whence by (2.1),

$$
\|x\| \leq \frac{\theta}{(1-\lambda) \gamma}\|T x\| \quad(x \in \mathscr{E}) .
$$

Now, fix $x, y \in \mathscr{E}$ with $x \perp^{\delta} y$. Hence $\|\langle x, y\rangle\| \leq \delta\|x\|\|y\|$. By (i) and (ii), we get

$$
\begin{aligned}
&\|\langle T x, T y\rangle\| \\
& \leq\|\langle T x, T y\rangle-\langle S x, S y\rangle\|+\|\langle S x, S y\rangle\| \\
& \leq\|\langle T x-S x, T y-S y\rangle+\langle T x-S x, S y\rangle+\langle S x, T y-S y\rangle\| \\
&+\theta^{2} \gamma^{2}\|\langle x, y\rangle\| \\
& \leq\|T x-S x\|\|T y-S y\|+\|T x-S x\|\|S y\|+\|S x\|\|T y-S y\| \\
&+\theta^{2} \gamma^{2} \delta\|x\|\|y\| \\
& \leq \lambda^{2}\|S x\|\|S y\|+2 \lambda\|S x\|\|S y\|+\theta^{2} \gamma^{2} \delta\|x\|\|y\| \\
& \leq\left(\lambda^{2}+2 \lambda\right)\|S x\|\|S y\|+\theta^{2} \gamma^{2} \delta \times \frac{\theta^{2}}{(1-\lambda)^{2} \gamma^{2}}\|T x\|\|T y\| \\
& \leq\left(\lambda^{2}+2 \lambda\right) \times \frac{1}{(1-\lambda)^{2}}\|T x\|\|T y\|+\frac{\theta^{4} \delta}{(1-\lambda)^{2}}\|T x\|\|T y\| \\
&= \frac{\lambda^{2}+2 \lambda+\theta^{4} \delta}{(1-\lambda)^{2}}\|T x\|\|T y\| .
\end{aligned}
$$

Thus $\|\langle T x, T y\rangle\| \leq \varepsilon\|T x\|\|T y\|$ and hence $T x \perp^{\varepsilon} T y$.

As a consequence, with $\theta=\sqrt[4]{\frac{\varepsilon}{\delta}}, \lambda=0$ and $S=T$, we have

Corollary 2.2. Let $\delta, \varepsilon \in[0,1)$. Let $\mathscr{E}$ and $\mathscr{F}$ be two inner product $\mathscr{A}$-modules and let $T: \mathscr{E} \longrightarrow \mathscr{F}$ be a nonzero linear mapping satisfying

$$
\sqrt{\frac{\delta}{\varepsilon}} \gamma^{2}\|\langle x, y\rangle\| \leq\|\langle T x, T y\rangle\| \leq \sqrt{\frac{\varepsilon}{\delta}} \gamma^{2}\|\langle x, y\rangle\|,
$$

for all $x, y \in \mathscr{E}$ with some $\gamma \in[[T],\|T\|]$. Then $T$ is a $(\delta, \varepsilon)$-orthogonality preserving mapping. 
It follows from the inequality in Corollary 2.2 that $x \perp y \Rightarrow T x \perp T y(x, y \in$ $\mathscr{E})$. In the following we give an example of $(\delta, \varepsilon)$-orthogonality preserving mapping between Hilbert $C^{*}$-modules.

Example 2.3. Let $0<\delta \leq \varepsilon<1$ and let $\mathscr{E}$ and $\mathscr{F}$ be two inner product $\mathscr{A}$-modules. We define $T: \mathscr{E} \longrightarrow \mathscr{F}$ by $T x=\sqrt{\frac{\varepsilon}{\delta}} x$. Suppose that $x, y \in \mathscr{E}$ satisfies $x \perp^{\delta} y$. Hence $\|\langle x, y\rangle\| \leq \delta\|x\|\|y\|$. Therefore, we get

$$
\begin{aligned}
\|\langle T x, T y\rangle\| & =\frac{\varepsilon}{\delta}\|\langle x, y\rangle\| \leq \varepsilon\|x\|\|y\|=\delta\left\|\sqrt{\frac{\varepsilon}{\delta}} x\right\|\left\|\sqrt{\frac{\varepsilon}{\delta}} y\right\| \\
& =\delta\|T x\|\|T y\| \leq \varepsilon\|T x\|\|T y\| .
\end{aligned}
$$

Thus $T x \perp^{\varepsilon} T y$. This shows that $T$ is a $(\delta, \varepsilon)$-orthogonality preserving mapping. In addition, if we consider $T x=\sqrt{\frac{\varepsilon}{\delta}}\|x\| x$, then for all $x, y \in \mathscr{E}$, the condition $x \perp^{\delta} y$ implies $T x \perp^{\varepsilon} T y$ but $T$ is not linear.

For inner product $\mathscr{A}$-module $\mathscr{E}$ we define the relation which is connected with the notion of angle. Fix $\delta, \varepsilon \in[0,1)$ and $c \in \mathscr{A}$ with $\|c\|<1$. Let us say $\angle_{c}^{\delta}$ if $\|\langle x, y\rangle-\| x\|\| y\|c\| \leq \delta\|x\|\|y\|$. A mapping $T: \mathscr{E} \longrightarrow \mathscr{F}$, where $\mathscr{E}$ and $\mathscr{F}$ are inner product $\mathscr{A}$-modules, is called $(\delta, \varepsilon, c)$-angle preserving, if $x \angle_{c}^{\delta} y \Rightarrow T x \angle_{c}^{\varepsilon} T y(x, y \in \mathscr{E})$. It is easy to see that $T$ is a $(\delta, \varepsilon, 0)$-angle preserving mapping if and only if $T$ is $(\delta, \varepsilon)$-orthogonality preserving.

Theorem 2.4. Let $\mathscr{E}$ be a full Hilbert $\mathscr{A}$-module with $\mathbb{K}(\mathscr{H}) \subseteq \mathscr{A} \subseteq \mathbb{B}(\mathscr{H})$ such that $\operatorname{dim} \mathscr{H}>1$ and let a nonzero bounded linear mapping $S: \mathscr{E} \longrightarrow \mathscr{E}$ satisfy

$$
|\langle S x, S y\rangle|=\|S\|^{2}|\langle x, y\rangle| \quad(x, y \in \mathscr{E}) .
$$

Let $c \in \mathscr{A}$ with $\|c\|<1, \delta \in[0,1-\|c\|)$ and $\theta \in[0,1)$. If a linear mapping $T: \mathscr{E} \longrightarrow \mathscr{E}$ satisfies $\|T-S\| \leq \theta\|S\|$, then $T$ is $(\delta, \varepsilon, c)$-angle preserving, where $\varepsilon=\frac{\theta^{2}+2 \theta+\delta+\left(\theta^{2}-2 \theta-2\right)\|c\|}{(1-\theta)^{2}}$.

Proof. For $x=z$ and $y=z,(2.3)$ becomes $\|S z\|=\|S\|\|z\|$. This implies

$$
|\|T z\|-\|S\|\|z\||=|\|T z\|-\|S z\|| \leq\|T z-S z\| \leq\|T-S\|\|z\| \leq \theta\|S\|\|z\| .
$$

Thus

$$
\|T z\| \leq(1+\theta)\|S\|\|z\| \quad \text { and } \quad\|z\| \leq \frac{\|T z\|}{\|S\|(1-\theta)} \quad(z \in \mathscr{E}) .
$$

From (2.3) we have $\left|\left\langle\frac{S}{\|S\|} x, \frac{S}{\|S\|} y\right\rangle\right|=|\langle x, y\rangle|(x, y \in \mathscr{E})$. So $\frac{S}{\|S\|}$ preserves the absolute value of the $\mathscr{A}$-valued inner product on $\mathscr{E}$. By the Wigner's theorem 
[3, Theorem 1] there exist an $\mathscr{A}$-linear isometry $U: \mathscr{E} \longrightarrow \mathscr{E}$ and a phase function $\varphi: \mathscr{E} \longrightarrow \mathbb{C}$ (i.e. its values are of modulus 1 ) such that

$$
\frac{S}{\|S\|} z=\varphi(z) U z \quad(z \in \mathscr{E})
$$

Now, let $x, y \in \mathscr{E}$ and $x \angle_{c}^{\delta} y$. By (2.4), we get

$$
\|x\|\|y\|-\frac{1}{\|S\|^{2}}\|T x\|\|T y\| \leq \frac{1}{\|S\|^{2}}\left(\frac{1}{(1-\theta)^{2}}-1\right)\|T x\|\|T y\|
$$

and

$$
\frac{1}{\|S\|^{2}}\|T x\|\|T y\|-\|x\|\|y\| \leq \frac{1}{\|S\|^{2}}\left(1-\frac{1}{(1+\theta)^{2}}\right)\|T x\|\|T y\| .
$$

Sine $1-\frac{1}{(1+\theta)^{2}} \leq \frac{1}{(1-\theta)^{2}}-1=\frac{2 \theta-\theta^{2}}{(1-\theta)^{2}},(2.6)$ and (2.7) yield

$$
\left|\|x\|\|y\|-\frac{1}{\|S\|^{2}}\|T x\|\|T y\|\right| \leq \frac{2 \theta-\theta^{2}}{\|S\|^{2}(1-\theta)^{2}}\|T x\|\|T y\| .
$$

Further, by (2.5) we get

$$
\begin{aligned}
&\left\|\left\langle\frac{T}{\|S\|} x, \frac{T}{\|S\|} y\right\rangle-\langle\varphi(x) U x, \varphi(y) U y\rangle\right\| \\
&=\|\left\langle\frac{T}{\|S\|} x, \frac{T}{\|S\|^{2}} y\right\rangle-\left\langle\frac{S}{\|S\|} x, \frac{S}{\|S\|} y\right\rangle \\
&=\frac{1}{\|S\|^{2}}\|\langle T x-S x, T y-S y\rangle+\langle T x-S x, S y\rangle+\langle S x, T y-S y\rangle\| \\
& \leq \frac{1}{\|S\|^{2}}(\|T x-S x\|\|T y-S y\|+\|T x-S x\|\|S y\|+\|S x\|\|T y-S y\|) \\
& \leq \frac{1}{\|S\|^{2}}\left(\|T-S\|^{2}\|x\|\|y\|+\|T-S\|\|S\|\|x\|\|y\|\right. \\
&\quad+\|S\|\|T-S\|\|x\|\|y\|) \\
& \leq \frac{1}{\|S\|^{2}}\left(\theta^{2}\|S\|^{2}\|x\|\|y\|+\theta\|S\|^{2}\|x\|\|y\|+\theta\|S\|^{2}\|x\|\|y\|\right) \\
&=\left(\theta^{2}+2 \theta\right)\|x\|\|y\| \\
& \leq \frac{\theta^{2}+2 \theta}{\|S\|^{2}(1-\theta)^{2}}\|T x\|\|T y\| .
\end{aligned}
$$


Since $x \angle_{c}^{\delta} y$, we have $\|\langle x, y\rangle-\| x\|\| y\|c\| \leq \delta\|x\|\|y\|$ and so we obtain

$$
\begin{aligned}
& \|\langle\varphi(x) U x, \varphi(y) U y\rangle-\overline{\varphi(x)} \varphi(y)\| x\|\| y\|c\| \\
& =|\overline{\varphi(x)}||\varphi(y)|\left\|\left\langle U^{*} U x, y\right\rangle-\right\| x\|\| y\|c\| \\
& =\|\langle x, y\rangle-\| x\|\| y\|c\| \\
& \leq \delta\|x\|\|y\| \\
& \leq \frac{\delta}{\|S\|^{2}(1-\theta)^{2}}\|T x\|\|T y\| .
\end{aligned}
$$

From (2.8) it follows that

$$
\begin{gathered}
\|\overline{\varphi(x)} \varphi(y)\| x\|\| y\left\|c-\frac{\overline{\varphi(x)} \varphi(y)}{\|S\|^{2}}\right\| T x\|\| T y\|c\| \\
=|\overline{\varphi(x)}||\varphi(y)|\left|\|x\|\|y\|-\frac{1}{\|S\|^{2}}\|T x\|\|T y\|\right|\|c\| \\
\leq \frac{2 \theta-\theta^{2}}{\|S\|^{2}(1-\theta)^{2}}\|T x\|\|T y\|\|c\| .
\end{gathered}
$$

Also, notice that

$$
\begin{aligned}
& \left\|\frac{\overline{\varphi(x)} \varphi(y)}{\|S\|^{2}}\right\| T x\|\| T y\left\|c-\frac{1}{\|S\|^{2}}\right\| T x\|\| T y\|c\| \\
& \quad=\frac{1}{\|S\|^{2}}\|T x\|\|T y\||\overline{\varphi(x)} \varphi(y)-1|\|c\| \\
& \quad \leq \frac{1}{\|S\|^{2}}\|T x\|\|T y\|(\overline{\varphi(x)}|| \varphi(y) \mid+1)\|c\|=\frac{2}{\|S\|^{2}}\|T x\|\|T y\|\|c\| .
\end{aligned}
$$


Now, we observe that

$$
\begin{aligned}
\|\langle T x, T y\rangle-\| T x\|\| T y\|c\| \\
\leq\|S\|^{2}\left(\left\|\left\langle\frac{T}{\|S\|} x, \frac{T}{\|S\|} y\right\rangle-\langle\varphi(x) U x, \varphi(y) U y\rangle\right\|\right. \\
+\|\langle\varphi(x) U x, \varphi(y) U y\rangle-\overline{\varphi(x)} \varphi(y)\| x\|\| y\|c\| \\
\left.\quad+\left\|\frac{\overline{\varphi(x)} \varphi(y)\|x\|\|y\| c-\frac{\overline{\varphi(x)} \varphi(y)}{\|S\|^{2}}\|T x\|\|T y\| c \|}{\| \overline{\varphi(x)} \varphi(y)}\right\| T x\|\| T y\left\|c-\frac{1}{\|S\|^{2}}\right\| T x\|\| T y\|c\|\right) \\
\left.\quad+\|S\|^{2} \|\right) \\
\leq \\
\quad\|S\|^{2}\left(\frac{\theta^{2}+2 \theta}{\|S\|^{2}(1-\theta)^{2}}\|T x\|\|T y\|+\frac{\delta}{\|S\|^{2}(1-\theta)^{2}}\|T x\|\|T y\|\right. \\
\left.+\frac{2 \theta-\theta^{2}}{\|S\|^{2}(1-\theta)^{2}}\|T x\|\|T y\|\|c\|+\frac{2}{\|S\|^{2}}\|T x\|\|T y\|\|c\|\right) \\
=\frac{\theta^{2}+2 \theta+\delta+\left(\theta^{2}-2 \theta-2\right)\|c\|}{(1-\theta)^{2}}\|T x\|\|T y\| .
\end{aligned}
$$

Thus $\|\langle T x, T y\rangle-\| T x\|\| T y\|c\| \leq \varepsilon\|T x\|\|T y\|$ and hence $T x \angle_{c}^{\varepsilon} T y$.

As a consequence, with $c=0$, we have

Corollary 2.5. Let $\delta, \theta \in[0,1)$. Let $\mathscr{E}$ be a full Hilbert $\mathscr{A}$-module with $\mathbb{K}(\mathscr{H}) \subseteq \mathscr{A} \subseteq \mathbb{B}(\mathscr{H})$ such that $\operatorname{dim} \mathscr{H}>1$ and let a nonzero bounded linear mapping $S: \mathscr{E} \longrightarrow \mathscr{E}$ satisfying

$$
|\langle S x, S y\rangle|=\|S\|^{2}|\langle x, y\rangle| \quad(x, y \in \mathscr{E}) .
$$

If a linear mapping $T: \mathscr{E} \longrightarrow \mathscr{E}$ satisfies $\|T-S\| \leq \theta\|S\|$, then $T$ is $(\delta, \varepsilon)$ orthogonality preserving, where $\varepsilon=\frac{\theta^{2}+2 \theta+\delta}{(1-\theta)^{2}}$.

\section{Mappings PReserving APPROXimate orthogonality in Hilbert}

$$
C^{*} \text {-MODULES }
$$

In this section, we study $(\delta, \varepsilon)$-orthogonality preserving mappings between Hilbert $\mathscr{A}$-modules whenever $\mathbb{K}(\mathscr{H}) \subseteq \mathscr{A} \subseteq \mathbb{B}(\mathscr{H})$. To achieve our main result we prove first some auxiliary results. 
Proposition 3.1. Let $T: \mathscr{H} \longrightarrow \mathscr{K}$ be a $(\delta, \varepsilon)$-orthogonality preserving linear mapping. If $\eta, \zeta \in \mathscr{H}$ are orthogonal unit vectors, then

$$
\sqrt{\frac{(n+1)(1-\delta)(1-\varepsilon)}{n(1+\delta)(1+\varepsilon)}}\|T \zeta\| \leq\|T \eta\| \leq \sqrt{\frac{(n+1)(1-\delta)(1+\varepsilon)}{n(1+\delta)(1-\varepsilon)}}\|T \zeta\|
$$

for all $n \in \mathbb{N}$.

Proof. Let $n \in \mathbb{N}$. We have

$$
\begin{aligned}
\mid(\eta+ & \left.\sqrt{\frac{(n+1)(1-\delta)}{n(1+\delta)}} \zeta, \eta-\sqrt{\frac{(n+1)(1-\delta)}{n(1+\delta)}} \zeta\right) \mid \\
& =1-\frac{(n+1)(1-\delta)}{n(1+\delta)} \\
& \leq \delta\left[1+\frac{(n+1)(1-\delta)}{n(1+\delta)}\right] \\
& =\delta\left\|\eta+\sqrt{\frac{(n+1)(1-\delta)}{n(1+\delta)}} \zeta\right\| \eta-\sqrt{\frac{(n+1)(1-\delta)}{n(1+\delta)}} \zeta \| .
\end{aligned}
$$

So, we get $\eta+\sqrt{\frac{(n+1)(1-\delta)}{n(1+\delta)}} \zeta \perp^{\delta} \zeta-\sqrt{\frac{(n+1)(1-\delta)}{n(1+\delta)}} \zeta$. Since $T$ is a $(\delta, \varepsilon)$-orthogonality preserving mapping, we reach

$$
T \eta+\sqrt{\frac{(n+1)(1-\delta)}{n(1+\delta)}} T \zeta \perp^{\varepsilon} T \eta-\sqrt{\frac{(n+1)(1-\delta)}{n(1+\delta)}} T \zeta .
$$

Therefore,

$$
\begin{aligned}
& \left|\left(T \eta+\sqrt{\frac{(n+1)(1-\delta)}{n(1+\delta)}} T \zeta, T \eta-\sqrt{\frac{(n+1)(1-\delta)}{n(1+\delta)}} T \zeta\right)\right| \\
& \quad \leq \varepsilon\left\|T \eta+\sqrt{\frac{(n+1)(1-\delta)}{n(1+\delta)}} T \zeta\right\| T \eta-\sqrt{\frac{(n+1)(1-\delta)}{n(1+\delta)}} T \zeta \|,
\end{aligned}
$$

whence

$$
\begin{aligned}
& \left(\|T \eta\|^{2}-\frac{(n+1)(1-\delta)}{n(1+\delta)}\|T \zeta\|^{2}\right)^{2}+4\left[\operatorname{Im}\left(T \eta, \sqrt{\frac{(n+1)(1-\delta)}{n(1+\delta)}} T \zeta\right)\right]^{2} \\
& \leq \varepsilon^{2}\left(\left(\|T \eta\|^{2}+\frac{(n+1)(1-\delta)}{n(1+\delta)}\|T \zeta\|^{2}\right)^{2}\right. \\
& \left.\quad-4\left[\operatorname{Re}\left(T \eta, \sqrt{\frac{(n+1)(1-\delta)}{n(1+\delta)}} T \zeta\right)\right]^{2}\right) .
\end{aligned}
$$


It follows that

$$
\left|\|T \eta\|^{2}-\frac{(n+1)(1-\delta)}{n(1+\delta)}\|T \zeta\|^{2}\right| \leq \varepsilon\left(\|T \eta\|^{2}+\frac{(n+1)(1-\delta)}{n(1+\delta)}\|T \zeta\|^{2}\right),
$$

or equivalently,

$$
\sqrt{\frac{(n+1)(1-\delta)(1-\varepsilon)}{n(1+\delta)(1+\varepsilon)}}\|T \zeta\| \leq\|T \eta\| \leq \sqrt{\frac{(n+1)(1-\delta)(1+\varepsilon)}{n(1+\delta)(1-\varepsilon)}}\|T \zeta\| .
$$

Corollary 3.2. Let $T: \mathscr{H} \longrightarrow \mathscr{K}$ be a $(\delta, \varepsilon)$-orthogonality preserving mapping. If $\eta, \zeta \in \mathscr{H} \backslash\{0\}$ are orthogonal vectors, then

$$
\sqrt{\frac{(1-\delta)(1-\varepsilon)}{(1+\delta)(1+\varepsilon)}}\|T \zeta\|\|\eta\| \leq\|T \eta\|\|\zeta\| \leq \sqrt{\frac{(1-\delta)(1+\varepsilon)}{(1+\delta)(1-\varepsilon)}}\|T \zeta\|\|\eta\| .
$$

Theorem 3.3. Let $T: \mathscr{H} \longrightarrow \mathscr{K}$ be a $(\delta, \varepsilon)$-orthogonality preserving mapping. Then $T$ is injective, continuous and satisfies

$$
\frac{1}{\theta} \gamma\|\eta\| \leq\|T \eta\| \leq \theta \gamma\|\eta\|
$$

for all $\eta \in \mathscr{H}, \gamma \in[[T],\|T\|]$ and $\theta=\sqrt{\frac{(1-\delta)(1+\varepsilon)}{(1+\delta)(1-\varepsilon)}+2 \varepsilon \sqrt{\frac{(1-\delta)(1+\varepsilon)}{(1+\delta)(1-\varepsilon)}}}$.

Proof. Let $\eta, \zeta \in \mathscr{H} \backslash\{0\}$. Choose $\eta_{1}, \eta_{2} \in \mathscr{H} \backslash\{0\}$ such that

$$
\eta=\eta_{1}+\eta_{2}, \quad \eta_{1} \in\{\lambda \zeta: \lambda \in \mathbb{C}\}, \quad\left|\left(\eta_{1}, \eta_{2}\right)\right|=0 \leq \delta\left\|\eta_{1}\right\|\left\|\eta_{2}\right\|,
$$

whence

$$
\|\eta\|^{2}=\left\|\eta_{1}\right\|^{2}+\left\|\eta_{2}\right\|^{2}, \quad\left\|\eta_{1}\right\| \leq\|\eta\|, \quad\left\|\eta_{2}\right\| \leq\|\eta\| .
$$

By Corollary 3.2, we get

$$
\sqrt{\frac{(1-\delta)(1-\varepsilon)}{(1+\delta)(1+\varepsilon)}}\left\|T \eta_{1}\right\|\left\|\eta_{2}\right\| \leq\left\|T \eta_{2}\right\|\left\|\eta_{1}\right\| \leq \sqrt{\frac{(1-\delta)(1+\varepsilon)}{(1+\delta)(1-\varepsilon)}}\left\|T \eta_{1}\right\|\left\|\eta_{2}\right\|
$$


So, we reach

$$
\begin{aligned}
& \|T \eta\|^{2}=\left\|T \eta_{1}\right\|^{2}+2 \operatorname{Re}\left(T \eta_{1}, T \eta_{2}\right)+\left\|T \eta_{2}\right\|^{2} \quad\left(\text { since } \eta=\eta_{1}+\eta_{2}\right) \\
& \leq\left\|T \eta_{1}\right\|^{2}+2\left|\left(T \eta_{1}, T \eta_{2}\right)\right|+\left\|T \eta_{2}\right\|^{2} \\
& \text { ( since } \frac{\left\|T \eta_{1}\right\|}{\left\|\eta_{1}\right\|}=\frac{\|T \zeta\|}{\|\zeta\|},\left|\left(\eta_{1}, \eta_{2}\right)\right| \leq \delta\left\|\eta_{1}\right\|\left\|\eta_{2}\right\| \text {, } \\
& T \text { is a }(\delta, \varepsilon) \text {-orthogonality preserving mapping and (3.3)) } \\
& \leq \frac{\|T \zeta\|^{2}}{\|\zeta\|^{2}}\left\|\eta_{1}\right\|^{2}+2 \varepsilon\left\|T \eta_{1}\right\|\left\|T \eta_{2}\right\|+\frac{(1-\delta)(1+\varepsilon)}{(1+\delta)(1-\varepsilon)} \frac{\left\|T \eta_{1}\right\|^{2}}{\left\|\eta_{1}\right\|^{2}}\left\|\eta_{2}\right\|^{2} \\
& \text { ( by (3.2), (3.3) and since } \frac{\left\|T \eta_{1}\right\|}{\left\|\eta_{1}\right\|}=\frac{\|T \zeta\|}{\|\zeta\|} \text { ) } \\
& \leq \frac{\|T \zeta\|^{2}}{\|\eta\|^{2}}\left(\|\eta\|^{2}-\left\|\eta_{2}\right\|^{2}\right)+2 \varepsilon \sqrt{\frac{(1-\delta)(1+\varepsilon)}{(1+\delta)(1-\varepsilon)}}\left\|T \eta_{1}\right\|^{2} \times \frac{\left\|\eta_{2}\right\|}{\left\|\eta_{1}\right\|} \\
& +\frac{(1-\delta)(1+\varepsilon)}{(1+\delta)(1-\varepsilon)} \frac{\|T \zeta\|^{2}}{\|\zeta\|^{2}}\left\|\eta_{2}\right\|^{2} \\
& \text { ( since } \frac{\left\|T \eta_{1}\right\|}{\left\|\eta_{1}\right\|}=\frac{\|T \zeta\|}{\|\zeta\|} \text { ) } \\
& \leq \frac{\|T \zeta\|^{2}}{\|\zeta\|^{2}}\|\eta\|^{2}+2 \varepsilon \sqrt{\frac{(1-\delta)(1+\varepsilon)}{(1+\delta)(1-\varepsilon)}} \frac{\|T \zeta\|^{2}}{\|\zeta\|^{2}}\left\|\eta_{1}\right\|\left\|\eta_{2}\right\| \\
& +\left(\frac{(1-\delta)(1+\varepsilon)}{(1+\delta)(1-\varepsilon)}-1\right) \frac{\|T \zeta\|^{2}}{\|\zeta\|^{2}}\left\|\eta_{2}\right\|^{2} \\
& \text { (since }\left\|\eta_{1}\right\| \leq\|\eta\| \text { and }\left\|\eta_{2}\right\| \leq\|\eta\| \text { ) } \\
& \leq \frac{\|T \zeta\|^{2}}{\|\zeta\|^{2}}\|\eta\|^{2}\left(1+2 \varepsilon \sqrt{\frac{(1-\delta)(1+\varepsilon)}{(1+\delta)(1-\varepsilon)}}+\left(\frac{(1-\delta)(1+\varepsilon)}{(1+\delta)(1-\varepsilon)}-1\right)\right) \\
& =\frac{\|T \zeta\|^{2}}{\|\zeta\|^{2}}\|\eta\|^{2}\left[\frac{(1-\delta)(1+\varepsilon)}{(1+\delta)(1-\varepsilon)}+2 \varepsilon \sqrt{\frac{(1-\delta)(1+\varepsilon)}{(1+\delta)(1-\varepsilon)}}\right] .
\end{aligned}
$$

Thus we have $\|T \eta\|^{2} \leq \frac{\|T \zeta\|^{2}}{\|\zeta\|^{2}}\|\eta\|^{2} \theta^{2}$ and hence $\frac{\|T \eta\|}{\|\eta\|} \leq \theta \frac{\|T \zeta\|}{\|\zeta\|}$. Since $\eta$ and $\zeta$ are arbitrary, we change the order to get $\frac{\|T \zeta\|}{\|\zeta\|} \leq \theta \frac{\|T \eta\|}{\|\eta\|}$ and finally $\frac{1}{\theta} \frac{\|T \zeta\|}{\|\zeta\|} \leq \frac{\|T \eta\|}{\|\eta\|} \leq$ $\theta \frac{\|T \zeta\|}{\|\zeta\|}$. Hence $T$ is continuous and $\frac{1}{\theta}\|T\| \leq \frac{\|T \eta\|}{\|\eta\|} \leq \theta[T]$.

Now, for all $\eta \in \mathscr{H}$ and for all $\gamma \in[[T],\|T\|]$, we reach

$$
\frac{1}{\theta} \gamma\|\eta\| \leq \frac{1}{\theta}\|T\|\|\eta\| \leq\|T \eta\| \leq \theta[T]\|\eta\| \leq \theta \gamma\|\eta\| .
$$

Thus $T$ is injective and $\frac{1}{\theta} \gamma\|\eta\| \leq\|T \eta\| \leq \theta \gamma\|\eta\|$.

The following lemma is a consequences of the discussion in the first section. 
Lemma 3.4. Let $\delta, \varepsilon \in[0,1)$. Let $\mathscr{E}$ be inner product $\mathscr{A}$-module with $\mathbb{K}(\mathscr{H}) \subseteq$ $\mathscr{A} \subseteq \mathbb{B}(\mathscr{H})$ and let $\mathscr{E}$ be any minimal projection. Then the following statements hold:

(i) $x, y \in \mathscr{E}_{e}$ are $\delta$-orthogonal in $\mathscr{E}_{e}$ if and only if they are $\delta$-orthogonal in $\mathscr{E}$.

(ii) If $T: \mathscr{E} \longrightarrow \mathscr{F}$ is an $\mathscr{A}$-linear $(\delta, \varepsilon)$-orthogonality preserving mapping, then $T_{e}:=\left.T\right|_{\mathscr{E}_{e}}: \mathscr{E}_{e} \longrightarrow \mathscr{F}_{e}$ is a linear $(\delta, \varepsilon)$-orthogonality preserving mapping.

Proof. (i) Let $x, y \in \mathscr{E}_{e}$. Then

$$
\begin{aligned}
x \perp^{\delta} y \text { in } \mathscr{E}_{e} & \Leftrightarrow|(x, y)| \leq \delta\|x\|_{\mathscr{E}_{e}}\|y\|_{\mathscr{E}_{e}} \Leftrightarrow\|\langle x, y\rangle\| \leq \delta\|x\|_{\mathscr{E}}\|y\|_{\mathscr{E}} \\
& \Leftrightarrow x \perp^{\delta} y \text { in } \mathscr{E} .
\end{aligned}
$$

(ii) Let $x \perp^{\delta} y$ in $\mathscr{E}_{e}$. By (i), $x \perp^{\delta} y$ in $\mathscr{E}$. Since $T$ is $(\delta, \varepsilon)$-orthogonality preserving, hence $T x \perp^{\varepsilon} T y$ in $\mathscr{F}$. So, by (i), $T_{e} x \perp^{\varepsilon} T_{e} y$ in $\mathscr{F}_{e}$. Thus $T_{e}$ is a linear $(\delta, \varepsilon)$-orthogonality preserving mapping.

A part of the following lemma can be found in [9, Proposition 3.3]. We, however, prove it for the sake of completeness.

Proposition 3.5. Let $\mathscr{E}, \mathscr{F}$ be inner product $\mathscr{A}$-modules with $\mathbb{K}(\mathscr{H}) \subseteq \mathscr{A} \subseteq$ $\mathbb{B}(\mathscr{H})$ and $T: \mathscr{E} \longrightarrow \mathscr{F}$ be an $\mathscr{A}$-linear mapping. Suppose that $T_{e}:=\left.T\right|_{\mathscr{E}_{e}}$ : $\mathscr{E}_{e} \longrightarrow \mathscr{F}_{e}$ for some minimal projection $\mathscr{E}$, such that $0<\left[T_{e}\right] \leq\left\|T_{e}\right\|<\infty$. Then

(i) $[T]=\left[T_{e}\right]$.

(ii) $\|T\|=\left\|T_{e}\right\|$.

Proof. (i) Let $e=\zeta \otimes \zeta, f=\eta \otimes \eta$ be minimal projections and let $u=\eta \otimes \zeta$. We have

$$
\begin{aligned}
e\langle T u, T u\rangle e & =\langle T(u e), T(u e)\rangle=(T(u e), T(u e)) e \\
& =\|T(u e)\|_{\mathscr{E}_{f}}^{2} e \geq\left[T_{e}\right]^{2}\|u e\|^{2} e=\left[T_{e}\right]^{2}\|(\eta \otimes \zeta)(\zeta \otimes \zeta)\|^{2} e \\
& =\left[T_{e}\right]^{2}\|\| \zeta\left\|^{2} \eta \otimes \zeta\right\|^{2} e=\left[T_{e}\right]^{2}\|u\|^{2} e .
\end{aligned}
$$

Hence

$$
\left[T_{e}\right]^{2}\|u\|^{2} \leq\|e\langle T u, T u\rangle e\| \leq \sup \{\|e\langle T u, T u\rangle e\|:\|e\|=1\}=\|T u\|^{2} .
$$


Hence $\left[T_{e}\right]\|u\| \leq\|T u\|$, which shows $\left[T_{e}\right] \leq[T]$. Since $\left[T_{e}\right] \geq[T]$, thus we reach $\left[T_{e}\right]=[T]$.

(ii) The proof is similar to (i).

We are now in a position to establish one of our main results. In fact, in the sequel we provide a version of Theorem 3.3 in the setting of inner product $C^{*}$-modules.

Theorem 3.6. Let $\delta, \varepsilon \in[0,1)$. Let $\mathscr{E}, \mathscr{F}$ be inner product $\mathscr{A}$-modules with $\mathbb{K}(\mathscr{H}) \subseteq \mathscr{A} \subseteq \mathbb{B}(\mathscr{H})$ and let $T: \mathscr{E} \longrightarrow \mathscr{F}$ be a nonzero $\mathscr{A}$-linear $(\delta, \varepsilon)$ orthogonality preserving mapping. Then

(i) $0<[T] \leq\|T\|<\infty$.

(ii) $\frac{1}{\theta^{2}} \gamma^{2}\langle x, x\rangle \leq\langle T x, T x\rangle \leq \theta^{2} \gamma^{2}\langle x, x\rangle$

for all $x \in \mathscr{E}, \gamma \in[[T],\|T\|]$ and $\theta=\sqrt{\frac{(1-\delta)(1+\varepsilon)}{(1+\delta)(1-\varepsilon)}+2 \varepsilon \sqrt{\frac{(1-\delta)(1+\varepsilon)}{(1+\delta)(1-\varepsilon)}}}$.

(iii) $\left\|\langle T x, T y\rangle-\gamma^{2}\langle x, y\rangle\right\| \leq 4\left(1-\frac{1}{\theta^{2}}\right) \min \left\{\gamma^{2}\|x\|\|y\|,\|T x\|\|T y\|\right\}$

for all $x, y \in \mathscr{E}$ and for all $\gamma \in[[T],\|T\|]$.

Proof. Let $e=\eta \otimes \eta$ be a minimal projection. From Lemma 3.4 it follows that $T_{e}:=\left.T\right|_{\mathscr{E}_{e}}: \mathscr{E}_{e} \longrightarrow \mathscr{F}_{e}$ is a linear $(\delta, \varepsilon)$-orthogonality preserving mapping. Hence Theorem 3.3 implies $T_{e}$ is injective, $0<\left[T_{e}\right] \leq\left\|T_{e}\right\|<\infty$ and satisfies

$$
\frac{1}{\theta} \gamma\|x e\| \leq\left\|T_{e}(x e)\right\| \leq \theta \gamma\|x e\|
$$

for all $x \in \mathscr{E}, \gamma \in\left[\left[T_{e}\right],\left\|T_{e}\right\|\right]$ and $\theta=\sqrt{\frac{(1-\delta)(1+\varepsilon)}{(1+\delta)(1-\varepsilon)}+2 \varepsilon \sqrt{\frac{(1-\delta)(1+\varepsilon)}{(1+\delta)(1-\varepsilon)}}}$. Thus by Proposition 3.5, $0<[T] \leq\|T\|<\infty$ and it follows from (3.4) that

$$
\frac{1}{\theta^{2}} \gamma^{2}(x e, x e) \leq\left(T_{e}(x e), T_{e}(x e)\right) \leq \theta^{2} \gamma^{2}(x e, x e)
$$

or equivalently,

$$
\left(\frac{1}{\theta^{2}} \gamma^{2}\langle x, x\rangle \eta, \eta\right) \leq(\langle T x, T x\rangle \eta, \eta) \leq\left(\theta^{2} \gamma^{2}\langle x, x\rangle \eta, \eta\right)
$$

Now (3.5) gives

$$
\frac{1}{\theta^{2}} \gamma^{2}\langle x, x\rangle \leq\langle T x, T x\rangle \leq \theta^{2} \gamma^{2}\langle x, x\rangle
$$

for all $x \in \mathscr{E}$ and for all $\gamma \in[[T],\|T\|]$. Using the polar identity, we obtain

$$
\begin{aligned}
\left\|\langle T x, T y\rangle-\gamma^{2}\langle x, y\rangle\right\| & \leq \frac{1}{4} \times 4\left(1-\frac{1}{\theta^{2}}\right) \gamma^{2}(\|x\|+\|y\|)^{2} \\
& \leq 2\left(1-\frac{1}{\theta^{2}}\right) \gamma^{2}\left(\|x\|^{2}+\|y\|^{2}\right)
\end{aligned}
$$


Applying (3.7) for vectors $\frac{x}{\|x\|}$ and $\frac{y}{\|y\|}$, we get

$$
\left\|\left\langle T\left(\frac{x}{\|x\|}\right), T\left(\frac{y}{\|y\|}\right)\right\rangle-\gamma^{2}\left\langle\frac{x}{\|x\|}, \frac{y}{\|y\|}\right\rangle\right\| \leq 4\left(1-\frac{1}{\theta^{2}}\right) \gamma^{2}
$$

or equivalently,

$$
\left\|\langle T x, T y\rangle-\gamma^{2}\langle x, y\rangle\right\| \leq 4\left(1-\frac{1}{\theta^{2}}\right) \gamma^{2}\|x\|\|y\| .
$$

Furthermore (3.6) implies

$$
\frac{1}{\theta^{2}} \frac{1}{\gamma^{2}}\langle T x, T x\rangle \leq\langle x, x\rangle \leq \theta^{2} \frac{1}{\gamma^{2}}\langle T x, T x\rangle .
$$

Similar to (3.9), by (3.10) we reach

$$
\left\|\langle T x, T y\rangle-\gamma^{2}\langle x, y\rangle\right\| \leq 4\left(1-\frac{1}{\theta^{2}}\right)\|T x\|\|T y\|,
$$

for all $x, y \in \mathscr{E}$ and for all $\gamma \in[[T],\|T\|]$. Thus, by (3.9) and (3.11), (iii) follows.

Next we obtain a sufficient condition for an $\mathscr{A}$-linear mapping to be $(\delta, \varepsilon)$ orthogonality preserving.

Corollary 3.7. Let $\delta, \varepsilon \in[0,1)$. Let $\mathscr{E}, \mathscr{F}$ be inner product $\mathscr{A}$-modules with $\mathbb{K}(\mathscr{H}) \subseteq \mathscr{A} \subseteq \mathbb{B}(\mathscr{H})$ and let $T: \mathscr{E} \longrightarrow \mathscr{F}$ be a nonzero $\mathscr{A}$-linear such that

$$
\begin{aligned}
\frac{2 \delta}{\sqrt{(4-\varepsilon)^{2}+16 \delta}-(4-\varepsilon)} \gamma^{2}\langle x, x\rangle & \leq\langle T x, T x\rangle \\
& \leq \frac{\sqrt{(4-\varepsilon)^{2}+16 \delta}-(4-\varepsilon)}{2 \delta} \gamma^{2}\langle x, x\rangle
\end{aligned}
$$

for all $x \in \mathscr{E}$ and for some $\gamma \in[[T],\|T\|]$. Then $T$ is $(\delta, \varepsilon)$-orthogonality preserving.

Proof. Let $x, y \in \mathscr{E}$ and $x \perp^{\delta} y$. Then $\|\langle x, y\rangle\| \leq \delta\|x\|\|y\|$. As in the proof of Theorem 3.6 (iii) we have

$$
\left\|\langle T x, T y\rangle-\gamma^{2}\langle x, y\rangle\right\| \leq 4\left(1-\frac{2 \delta}{\sqrt{(4-\varepsilon)^{2}+16 \delta}-(4-\varepsilon)}\right)\|T x\|\|T y\| .
$$


Hence

$$
\begin{aligned}
& \|\langle T x, T y\rangle\| \\
& \leq\left\|\langle T x, T y\rangle-\gamma^{2}\langle x, y\rangle\right\|+\gamma^{2}\|\langle x, y\rangle\| \\
& \leq 4\left(1-\frac{2 \delta}{\sqrt{(4-\varepsilon)^{2}+16 \delta}-(4-\varepsilon)}\right)\|T x\|\|T y\|+\gamma^{2} \delta\|x\|\|y\| \\
& \leq 4\left(1-\frac{2 \delta}{\sqrt{(4-\varepsilon)^{2}+16 \delta}-(4-\varepsilon)}\right)\|T x\|\|T y\| \\
& +\gamma^{2} \delta \frac{\sqrt{\sqrt{(4-\varepsilon)^{2}+16 \delta}-(4-\varepsilon)}}{\gamma \sqrt{2 \delta}}\|T x\| \\
& \frac{\sqrt{\sqrt{(4-\varepsilon)^{2}+16 \delta}-(4-\varepsilon)}}{\gamma \sqrt{2 \delta}}\|T y\| \\
& \leq\left[4\left(1-\frac{2 \delta}{\sqrt{(4-\varepsilon)^{2}+16 \delta}-(4-\varepsilon)}\right)+\frac{\sqrt{(4-\varepsilon)^{2}+16 \delta}-(4-\varepsilon)}{2}\right] \\
& \cdot\|T x\|\|T y\|=\varepsilon\|T x\|\|T y\| .
\end{aligned}
$$

Thus $T x \perp^{\varepsilon} T y$.

Let us quote a result from [17].

Lemma 3.8. [17, Theorem 3.4] Let $\delta, \varepsilon \in[0,1)$. Let $\mathscr{H}, \mathscr{K}$ be Hilbert spaces and let $T: \mathscr{H} \longrightarrow \mathscr{K}$ be a nonzero $(\delta, \varepsilon)$-orthogonality preserving mapping. Then $T$ satisfies $\theta\|T\|\|\xi\| \leq\|T \xi\|$ for all $\xi \in \mathscr{H}$, with $\theta=\sqrt{\frac{(1-\delta)(1+\varepsilon)}{(1+\delta)(1-\varepsilon)}}$.

Theorem 3.9. Let $\delta, \varepsilon \in[0,1)$. Let $\mathscr{E}, \mathscr{F}$ be Hilbert $\mathscr{A}$-modules with $\mathbb{K}(\mathscr{H}) \subseteq$ $\mathscr{A} \subseteq \mathbb{B}(\mathscr{H})$ and let $T: \mathscr{E} \longrightarrow \mathscr{F}$ be a nonzero $\mathscr{A}$-linear $(\delta, \varepsilon)$-orthogonality preserving mapping. Then

(i) $\frac{(1+\delta)(1-\varepsilon)}{(1-\delta)(1+\varepsilon)}\|T\|^{2}\langle x, x\rangle \leq\langle T x, T x\rangle \leq\|T\|^{2}\langle x, x\rangle$ for all $x \in \mathscr{E}$.

(ii) $\|\langle T x, T y\rangle-\| T\left\|^{2}\langle x, y\rangle\right\| \leq \frac{4(\varepsilon-\delta)}{(1-\delta)(1+\varepsilon)}\|T x\|\|T y\|$ for all $x, y \in \mathscr{E}$.

Proof. By Lemma 3.8, we have $\theta\|T\|\|\xi\| \leq\|T \xi\| \leq\|T\|\|\xi\| \leq \frac{1}{\theta}\|T\|\|\xi\|$ for all $\xi \in \mathscr{H}$, with $\theta=\sqrt{\frac{(1-\delta)(1+\varepsilon)}{(1+\delta)(1-\varepsilon)}}$. Thus the proof is similar to the proof of Theorem 3.6 and so we omit it.

Now, we are going to show some applications of the above theorems, which generalize some results from $[4,9,17,19,20]$. 
As a consequence of Theorem 3.6 and Theorem 3.9, we have the following result.

Corollary 3.10. Let $0 \leq \varepsilon<\delta<1$. Let $\mathscr{E}, \mathscr{F}$ be Hilbert $\mathscr{A}$-modules with $\mathbb{K}(\mathscr{H}) \subseteq \mathscr{A} \subseteq \mathbb{B}(\mathscr{H})$ and $T: \mathscr{E} \longrightarrow \mathscr{F}$ be an $\mathscr{A}$-linear $(\delta, \varepsilon)$-orthogonality preserving mapping. Then $T=0$.

Proof. We suppose, for a contradiction, that there is a nonzero $\mathscr{A}$-linear $(\delta, \varepsilon)$ orthogonality preserving mapping with $0 \leq \varepsilon<\delta<1$. According to Theorem 3.6 (i), $0<[T] \leq\|T\|<\infty$ and also by Theorem 3.9, we have $\frac{1}{\theta^{2}}\|T\|^{2}\langle x, x\rangle \leq$ $\langle T x, T x\rangle \leq\|T\|^{2}\langle x, x\rangle$ for all $x \in \mathscr{E}$, with $\theta=\sqrt{\frac{(1-\delta)(1+\varepsilon)}{(1+\delta)(1-\varepsilon)}}$. Since $\theta<1$, we obtain

$$
0<\|T\|^{2}\langle x, x\rangle<\frac{1}{\theta^{2}}\|T\|^{2}\langle x, x\rangle \leq\langle T x, T x\rangle \leq\|T\|^{2}\langle x, x\rangle
$$

for all $x \in \mathscr{E}$, a contradiction. Therefore, $T=0$.

Corollary 3.11. Let $\delta, \varepsilon \in[0,1)$. Let $\mathscr{E}, \mathscr{F}$ be Hilbert $\mathscr{A}$-modules with $\mathbb{K}(\mathscr{H}) \subseteq$ $\mathscr{A} \subseteq \mathbb{B}(\mathscr{H})$ and let for any $n \in \mathbb{N}, T_{n}: \mathscr{E} \longrightarrow \mathscr{F}$ be an $\mathscr{A}$-linear $(\delta, \varepsilon)$ orthogonality preserving mapping. If $T: \mathscr{E} \longrightarrow \mathscr{F}$ is a bounded linear mapping such that $T_{n} \rightarrow T$, then $T$ is $\varphi$-orthogonality preserving with $\varphi=\frac{4(\varepsilon-\delta)}{(1-\delta)(1+\varepsilon)}$.

Proof. Let $x, y \in \mathscr{E}$ and $x \perp y$. Hence for any $n \in \mathbb{N}$, by Theorem 3.9 (ii), we have $\left\|\left\langle T_{n} x, T_{n} y\right\rangle\right\| \leq \varphi\left\|T_{n} x\right\|\left\|T_{n} y\right\|$, for all $x, y \in \mathscr{E}$, with $\varphi=\frac{4(\varepsilon-\delta)}{(1-\delta)(1+\varepsilon)}$. Thus

$$
\begin{aligned}
\|\langle T x, T y\rangle\| \leq & \left\|\langle T x, T y\rangle-\left\langle T_{n} x, T y\right\rangle\right\|+\left\|\left\langle T_{n} x, T y\right\rangle-\left\langle T_{n} x, T_{n} y\right\rangle\right\| \\
& +\left\|\left\langle T_{n} x, T_{n} y\right\rangle\right\| \\
\leq & \left\|T_{n}-T\right\|\|x\|\|T y\|+\left\|T_{n} x\right\|\left\|T-T_{n}\right\|\|y\| \\
& +\frac{4(\varepsilon-\delta)}{(1-\delta)(1+\varepsilon)}\left\|T_{n} x\right\|\left\|T_{n} y\right\| .
\end{aligned}
$$

Letting $n \rightarrow \infty$, we obtain $\|\langle T x, T y\rangle\| \leq \frac{4(\varepsilon-\delta)}{(1-\delta)(1+\varepsilon)}\|T x\|\|T y\|$, which is nothing else but $T x \perp^{\varphi} T y$.

Taking $\mathscr{E}=\mathscr{F}$ and $T=i d$, one obtains, from Theorem 3.9 the following result.

Corollary 3.12. Let $\delta, \varepsilon, \vartheta \in[0,1)$. Let $\mathscr{E}$ be a Hilbert $\mathscr{A}$-module with $\mathbb{K}(\mathscr{H}) \subseteq \mathscr{A} \subseteq \mathbb{B}(\mathscr{H})$ and let $\langle,\rangle_{1}$ and $\langle,\rangle_{2}$ be two $\mathscr{A}$-valued inner products on $\mathscr{E}$. If $\perp_{1}^{\delta} \subseteq \perp_{2}{ }^{\varepsilon}$, i.e., if $\left\|\langle x, y\rangle_{1}\right\| \leq \delta\|x\|_{1}\|y\|_{1} \Rightarrow\left\|\langle x, y\rangle_{2}\right\| \leq \varepsilon\|x\|_{2}\|y\|_{2}$ for all $x, y \in \mathscr{E}$, then there exists $\gamma>0$ such that 
(i) $\frac{\gamma}{\theta^{2}}\langle x, x\rangle_{1} \leq\langle x, x\rangle_{2} \leq \gamma\langle x, x\rangle_{1}$ for all $x \in \mathscr{E}$, with $\theta=\sqrt{\frac{(1-\delta)(1+\varepsilon)}{(1+\delta)(1-\varepsilon)}}$.

(ii) $\left\|\langle x, y\rangle_{2}-\gamma\langle x, y\rangle_{1}\right\| \leq \varphi \min \left\{\gamma\|x\|_{1}\|y\|_{1},\|x\|_{2}\|y\|_{2}\right\}$ for all $x, y \in \mathscr{E}$, with $\varphi=\frac{4(\varepsilon-\delta)}{(1-\delta)(1+\varepsilon)}$.

(iii) $\perp_{2}{ }^{\vartheta} \subseteq \perp_{1}{ }^{\nu}$, with $\nu=\vartheta+\frac{4(\varepsilon-\delta)}{(1-\delta)(1+\varepsilon)}$, which makes sense if $\nu<1$, i.e., for sufficiently small $\delta, \varepsilon$ and $\vartheta$.

Next we obtain some characterizations of the orthogonality preserving mappings in Hilbert $\mathscr{A}$-modules.

Corollary 3.13. Let $\varepsilon \in[0,1)$. Let $\mathscr{E}, \mathscr{F}$ be Hilbert $\mathscr{A}$-modules with $\mathbb{K}(\mathscr{H}) \subseteq$ $\mathscr{A} \subseteq \mathbb{B}(\mathscr{H})$. For a nonzero $\mathscr{A}$-linear mapping $T: \mathscr{E} \longrightarrow \mathscr{F}$ the following statements are equivalent:

(i) There exists $\gamma>0$ such that $\|T x\|=\gamma\|x\|$ for all $x \in \mathscr{E}$.

(ii) $T$ is injective and $\frac{\langle T x, T y\rangle}{\|T x\|\|T y\|}=\frac{\langle x, y\rangle}{\|x\|\|y\|}$ for all $x, y \in \mathscr{E} \backslash\{0\}$.

(iii) $|x|=|y| \Rightarrow|T x|=|T y|$ for all $x, y \in \mathscr{E}$.

(iv) $|x| \leq|y| \Rightarrow|T x| \leq|T y|$ for all $x, y \in \mathscr{E}$.

(v) $T$ is strongly orthogonality preserving.

(vi) $T$ is orthogonality preserving.

(vii) $T$ is strongly $(\varepsilon, \varepsilon)$-orthogonality preserving.

(viii) $T$ is $(\varepsilon, \varepsilon)$-orthogonality preserving.

Proof. It follows from Theorem (4.6) and Corollary (4.11) of [19] we have (i) $\Leftrightarrow(\mathrm{ii}) \Leftrightarrow(\mathrm{iii}) \Leftrightarrow(\mathrm{iv}) \Leftrightarrow(\mathrm{v}) \Leftrightarrow(\mathrm{vi})$.

(ii) $\Rightarrow$ (vii) and (vii) $\Rightarrow$ (viii) are trivial.

To prove (viii) $\Rightarrow(\mathrm{i})$, let $\delta:=\varepsilon$. From Theorem 3.9 we obtain

$$
\frac{(1+\varepsilon)(1-\varepsilon)}{(1-\varepsilon)(1+\varepsilon)}\|T\|^{2}\langle x, x\rangle \leq\langle T x, T x\rangle \leq\|T\|^{2}\langle x, x\rangle
$$

for all $x \in \mathscr{E}$. Thus $\langle T x, T x\rangle=\|T\|^{2}\langle x, x\rangle$ for all $x \in \mathscr{E}$.

The following example shows that conditions (iii)-(viii) in Corollary 3.13 are not equivalent to conditions (i)-(ii), even in the case $\varepsilon=0$, in an arbitrary Hilbert $\mathscr{A}$-module.

Example 3.14. Following [19, Example 4.7], let $\Omega$ be a locally compact Hausdorff space. Let us take $\mathscr{E}=\mathscr{F}=C_{0}(\Omega)$, the $C^{*}$-algebra of all continuous complex-valued functions vanishing at infinity on $\Omega$. For a nonzero function 
$f_{0} \in C_{0}(\Omega)$, suppose that $T: C_{0}(\Omega) \longrightarrow C_{0}(\Omega)$ is given by $T(g)=f_{0} g$. Obviously $T$ is $C_{0}(\Omega)$-linear and satisfies conditions (iii)-(viii) but need not satisfies conditions (i)-(ii). Indeed, if there exists $\gamma>0$ such that $\|T(g)\|=\gamma\|g\|$ for all $g \in C_{0}(\Omega)$, then $\frac{1}{\gamma^{2}} \overline{f_{0}} f_{0} g=g$ for all $g \in C_{0}(\Omega)$ and hence, $\frac{1}{\gamma^{2}} \overline{f_{0}} f_{0}$ is the identity in $C_{0}(\Omega)$, which is a contradiction.

Note that the assumption of $\mathscr{A}$-linearity, even in the case $\varepsilon=0$ and $\mathscr{E}=$ $\mathscr{F}=\mathscr{A}=\mathbb{B}(\mathscr{H})$, is necessary in Corollary 3.13 as one can see from the following example.

Example 3.15. Let $\mathscr{E}=\mathscr{F}=\mathbb{B}(\mathscr{H})$ and let $P \in \mathbb{B}(\mathscr{H})$ be a nontrivial projection. Then there exists $S_{1} \in \mathbb{B}(\mathscr{H})$ such that $S_{1} P \neq P S_{1}$. Hence there exists $S_{2} \in \mathbb{B}(\mathscr{H})$ such that $S_{2}\left(S_{1} P-P S_{1}\right) \neq 0$. Now, the mapping $T: \mathbb{B}(\mathscr{H}) \longrightarrow \mathbb{B}(\mathscr{H})$ defined by $T(S)=S P$ is orthogonality preserving. Since $T\left(S_{2} S_{1}\right)-T\left(S_{2}\right) S_{1}=S_{2}\left(S_{1} P-P S_{1}\right) \neq 0$, so $T$ is not $\mathbb{B}(\mathscr{H})$-linear. But $T$ does not satisfy (i). Indeed, if there exists $\gamma>0$ such that $\|T(S)\|=\gamma\|S\|$ for all $S \in \mathbb{B}(\mathscr{H})$, then for $S=P$ we get $\gamma=1$. But $P$ is a nontrivial projection and we obtain a contradiction; see [9, Example 3.2].

Corollary 3.16. Let $\delta, \varepsilon \in[0,1)$ and let $\mathscr{E}, \mathscr{F}$ be Hilbert $\mathscr{A}$-modules. The following statements hold:

(i) If $S: \mathscr{E} \longrightarrow \mathscr{E}$ is a linear $(\delta, \delta)$-orthogonality preserving mapping and $T$ is $(\delta, \varepsilon)$-orthogonality preserving, then $T S$ is linear $(\delta, \varepsilon)$-orthogonality preserving mapping.

(ii) If $S: \mathscr{F} \longrightarrow \mathscr{F}$ is a nonzero $\mathscr{A}$-linear $(\varepsilon, \varepsilon)$-orthogonality preserving mapping with $\mathbb{K}(\mathscr{H}) \subseteq \mathscr{A} \subseteq \mathbb{B}(\mathscr{H})$ and $T$ is an $\mathscr{A}$-linear $(\delta, \varepsilon)$ orthogonality preserving mapping, then $S T$ is $\mathscr{A}$-linear $(\delta, \varepsilon)$-orthogonality preserving.

Proof. The proof immediately follows from the definition of a $(\delta, \varepsilon)$-orthogonality preserving mapping and the equivalence (i) $\Leftrightarrow$ (iv) of Corollary 3.13.

Acknowledgements The authors would like to thank the referee for several useful comments.

\section{REFERENCES}

1. Lj. Arambašić and R. Rajić, A strong version of the Birkhoff-James orthogonality in Hilbert $C^{*}$-modules, Ann. Funct. Anal. 5 (2014), no. 1, 109-120. 
2. D. Bakić and B. Guljaš, Hilbert $C^{*}$-modules over $C^{*}$-algebras of compact operators, Acta Sci. Math. (Szeged) 68 (2002) 249-269.

3. D. Bakić and B. Guljaš, Wigners theorem in a class of Hilbert $C^{*}$-modules, J. Math. Phys. 44 (2003) 2186-2191.

4. J. Chmieliński, Linear mappings approximately preserving orthogonality, J. Math. Anal. Appl. 304 (2005), 158-169.

5. J. Chmieliński, Orthogonality equation with two unknown functions, Aequationes Math. 90 (2016), 11-23.

6. J. Chmieliński, R. Eukasik and P. Wójcik, On the stability of the orthogonality equation and the orthogonality-preserving property with two unknown functions, Banach J. Math. Anal. 10 (2016), no. 4, 828-847.

7. J. Chmieliński and P. Wójcik, Isosceles-orthogonality preserving property and its stability, Nonlinear Anal. 72 (2010), 1445-1453.

8. M. Frank, A. S. Mishchenko and A. A. Pavlov, Orthogonality-preserving, $C^{*}$-conformal and conformal module mappings on Hilbert $C^{*}$-modules, J. Funct. Anal. 260 (2011), 327-339.

9. D. Ilišević and A. Turnšek, Approximately orthogonality preserving mappings on $C^{*}$ modules, J. Math. Anal. Appl. 341 (2008), 298-308.

10. L. Kong and H. Cao, Stability of orthogonality preserving mapping and the orthogonality equation, J. Shaanxi Normal Univ. Nat. Sci. Ed. 36(5) (2008) 10-14.

11. C.-W. Leung, C.-K. Ng and N.-C. Wong, Linear orthogonality preservers of Hilbert $C^{*}$ modules over $C^{*}$-algebras with real rank zero, Proc. Amer. Math. Soc. 140 (2012), no. 9, 3151-3160.

12. R. Eukasik and P. Wójcik, Decomposition of two functions in the orthogonality equation, Aequationes Math. 90 (2016), 495-499.

13. V. M. Manuilov and E. V. Troitsky, Hilbert $C^{*}-$ modules, In: Translations of Mathematical Monographs. 226, American Mathematical Society, Providence, RI, 2005.

14. B. Mojškerc and A. Turnšek, Mappings approximately preserving orthogonality in normed spaces, Nonlinear Anal. 73 (2010), 3821-3831.

15. V. Pambuccian, A logical look at characterizations of geometric transformations under mild hypotheses, Indag. Math. (N.S.) 11 (2000), no. 3, 453-462.

16. A. Turnšek, On mappings approximately preserving orthogonality, J. Math. Anal. Appl. 336 (1) (2007), 625-631.

17. P. Wójcik, On certain basis connected with operator and its applications, J. Math. Anal. Appl. 423 (2) (2015), 1320-1329.

18. A. Zamani and M.S. Moslehian, Approximate Roberts orthogonality, Aequationes Math. 89 (2015), 529-541.

19. A. Zamani, M. S. Moslehian and M. Frank, Angle preserving mappings, Z. Anal. Anwend. 34 (2015), 485-500.

20. Y. Zhang, Y. Chen, D. Hadwin and L. Kong, AOP mappings and the distance to the scalar multiples of isometries, J. Math. Anal. Appl. 431 (2) (2015), 1275-1284. 
Department of Pure Mathematics, Ferdowsi University of Mashhad, P.O. Box 1159, MASHHAD 91775, Iran

E-mail address: moslehian@um.ac.ir, moslehian@member.ams.org; Zamani.ali85@yahoo.com 